Research Article

\title{
Joint Ordering and Pricing Decisions for New Repeat-Purchase Products
}

\author{
Xiang $\mathrm{Wu}^{1,2}$ and Jinlong Zhang ${ }^{1,2,3}$ \\ ${ }^{1}$ School of Management, Huazhong University of Science \& Technology, Wuhan 430074, China \\ ${ }^{2}$ Modern Information Management Center, Huazhong University of Science and Technology, 1037 Luoyu Road, Wuhan 430074, China \\ ${ }^{3}$ Collaborative Innovation Center for Modern Logistics and Business of Hubei, Wuhan Technology and Business University, \\ Wuhan 430065, China
}

Correspondence should be addressed to Xiang Wu; wuhsiang@hust.edu.cn

Received 28 March 2015; Accepted 28 June 2015

Academic Editor: Carlo Piccardi

Copyright (C) $2015 \mathrm{X}$. Wu and J. Zhang. This is an open access article distributed under the Creative Commons Attribution License, which permits unrestricted use, distribution, and reproduction in any medium, provided the original work is properly cited.

This paper studies ordering and pricing problems for new repeat-purchase products. We incorporate the repeat-purchase rate and price effects into the Bass model to characterize the demand pattern. We consider two decision models: (1) two-stage decision model, in which the sales division chooses a price to maximize the gross profit and the purchasing division determines an optimal ordering decision to minimize the total cost under a given demand subsequently, and (2) joint decision model, in which the firm makes ordering and pricing decisions simultaneously to maximize the profit. We combine the generalized Bass model with dynamic lot sizing model to formulate the joint decision model. We apply both models to a specific imported food provided by an online fresh produce retailer in Central China, solve them by Gaussian Random-Walk and Wagner-Whitin based algorithms, and observe three results. First, joint pricing and ordering decisions bring more significant profits than making pricing and ordering decisions sequentially. Second, a great initiative in adoption significantly increases price premium and profit. Finally, the optimal price shows a U-shape (i.e., decreases first and increases later) relationship and the profit increases gradually with the repeat-purchase rate when it is still not very high.

\section{Introduction}

When introducing a new repeat-purchase product into the market, the firm faces a complicated demand pattern. First, the new product diffuses in the market and more and more consumers begin to adopt the product. The Bass model assumes that consumers are influenced by two kinds of communication: mass media and word of mouth [1]. The first consumer group is only influenced by mass media (also called "external influence"), while the other group is only influenced by word of mouth (also called "internal influence"). The external influence reflects the initiative of consumers in adopting the new product, while the internal influence characterizes the imitation behavior in adoption. Second, the demand consists of two parts: the trial purchase from the new adopters and the repeat purchase from the existing adopters [2]. Finally, price decisions also influence how the product diffuses [3]. If the firm charges a lower price, it earns less for a unit product but harvests more sales volumes derived from the trial and repeat purchase. Thereby, the firm should get an in-depth understanding of the demand evolution and carefully choose the price level.

The firm can use dynamic lot sizing strategy to deal with dynamic demands derived from the diffusion process, that is, reviewing the inventory periodically and deciding whether to replenish products. The firm makes ordering decisions with regard to the demand at each period, which partly depends on the firm's pricing decisions. Thus, both the ordering and pricing decisions are essential for new repeatpurchase products. We consider two scenarios: (1) two-stage decision scenario, in which the sales division determines a price to maximize the gross profit and the purchasing division makes ordering decisions to minimize the total cost subsequently, and (2) joint decision scenario, in which the firm makes pricing and ordering decisions simultaneously to maximize the profit. In each scenario, we consider how 
the new product diffusion patterns and repeat-purchase rate influence ordering and pricing decisions. Usually, the ratio of the internal influence to the external influence has large variation for different products while the sum of these two influences does not [4]. Hence, we focus on the effects of the following two factors: the ratio of internal influence to the external influence and the repeat-purchase rate.

The remainder of the paper is organized as follows. We discuss the relevant literature in the next section. In Section 3, we use dynamic lot sizing model and generalized Bass model to formulate both two-stage and joint decision models. In Section 4, we give an upper and lower bound of the optimal price for both models and develop heuristic algorithms to solve them. We give a case with sensitivity analysis in Section 5 and make concluding remarks in Section 6.

\section{Literature Review}

Our study mainly relates to two research domains: the Bass model with price effects and joint inventory and pricing models.

The underlying premise of the Bass model lies in that the probability of adoption of a new product for nonadopters at each period linearly depends on two forces: innovators (or "external influence") and imitators (or "internal influence") [1]. The number of previous adopters positively influences the latter force but has no impact on the former one. The Bass model has both continuous and discrete forms, allowing us to examine product diffusion process under various circumstances $[1,5]$. Besides, pricing new products requires a thorough understanding of the complex dynamics associated with product diffusion process $[3,6]$. We adopt the discrete form of the Bass model and consider product diffusion in a much broad setting with dynamic lot sizing and pricing decisions. Very few studies combine the Bass model with inventory decisions, except Bilginer and Erhun [7] and Bhattacharya et al. [8].

This research also closely relates to the growing research stream on joint pricing and inventory management (see the review paper of Yano and Gilbert [9]). Scholars investigate joint decisions in various setting, such as perishable or substitutable products [10], noninstantaneous decaying items $[11,12]$, backorder [13], weather-sensitive demand [14], and demand uncertainty [15]. We contribute to this research stream by considering joint pricing and ordering decisions in the new repeat-purchase product setting.

\section{Ordering and Pricing Models}

3.1. Problem Description. We consider a firm selling a new repeat-purchase product in the following $T$ periods. The new repeat-purchase product has its own diffusion pattern characterized by the Bass model and also influenced by the price. Thus, the firm makes ordering and pricing decisions based on the understanding of new repeat-purchase product diffusion process.

This paper considers two models: (1) two-stage decision model, in which the sales division determines a price to maximize the gross profit and the purchasing division makes ordering decisions subsequently, and (2) joint decision model, in which the firm makes pricing and ordering decisions simultaneously to maximize the profit.

3.2. Assumptions and Denotations. We assume that the consumer purchases at most one unit of the new product at each period and uses the following denotations:
$T$ : total periods of the planning horizon;
$t$ : index for periods, $t=1, \ldots, T$;
$d(t)$ : demand at period $t$;
$x(t)$ : inventory at the end of period $t$;
$z(t)$ : order size at period $t$;
$v(t)$ : a binary indicator variable, $v(t)=1$ if an order occurs at period $t$ and 0 if not;
$n(t)$ : number of new adopters at period $t$;
$N(t)$ : cumulative number of adopters by period $t$;
$\lambda$ : repeat-purchase rate;
$k(t)$ : set-up cost at period $t$;
$c(t)$ : unit ordering cost at period $t$;
$h(t)$ : unit holding cost at period $t$;
$m$ : market potential for the new product, defined as the possible largest amount of consumers;
$p$ : coefficient of innovation;
$q$ : coefficient of imitation.

3.3. Demand. We use the discrete form of the Bass model with the price effect to describe how the new repeat-purchase product diffuses. The number of the adopters at period $t$ is $[3,5]$

$$
\begin{aligned}
& n(t) \\
& = \begin{cases}{\left[p+\frac{q}{m} N(t-1)\right][m-N(t-1)] e^{\beta\left(P / P_{0}-1\right)},} & t=2, \ldots, T \\
p m e^{\beta\left(P / P_{0}-1\right)}, & t=1 .\end{cases}
\end{aligned}
$$

The Bass model assumes that consumers are influenced by two kinds of communication: mass media and word of mouth. The first consumer group is only influenced by mass media (also called "external influence"), while the other group is only influenced by word of mouth (also called "internal influence"). Since $m-N(t)$ consumers still have not adopted the product by period $t$, the adoption rate at period $t$, $n(t) /[m-N(t)]$, consists of two parts: $p$ represents adoption from those who are only influenced by mass media and $q N(t-$ $1) / m$ represents adoption from those who are influenced by the existing adopters that make up a proportion of $N(t-$ $1) / m$. The last term, $e^{\beta\left(P / P_{0}-1\right)}$, characterizes the effect of price on product diffusion. When $P=P_{0}, e^{\beta\left(P / P_{0}-1\right)}=1$. In consequence, $p$ and $q$ represent the diffusion parameter given price $P=P_{0}$.

For $2 \leq t \leq T$, the demand at period $t$ consists of two parts: the trial purchase from new adopters and 
the repeat purchase from the cumulative adopters. We further assume that the price influences the trial purchase and repeat purchase in the same way. Thereby, the demand derived from repeat purchase would be $\lambda e^{\beta\left(P / P_{0}-1\right)} N(t-1)$. When offering very low prices, the firm makes at most all cumulative adopters purchase repeatedly. In consequence, the demand from repeat purchase is $\min \left\{1, \lambda e^{\beta\left(P / P_{0}-1\right)}\right\} N(t-1)$. Thus, the demand at period $t$ is

$$
\begin{aligned}
& d(t) \\
& = \begin{cases}n(t)+\min \left\{1, \lambda e^{\beta\left(P / P_{0}-1\right)}\right\} N(t-1), & t=2, \ldots, T \\
n(t), & t=1 .\end{cases}
\end{aligned}
$$

3.4. A Two-Stage Decision Model. In the first stage, the sales division determines an optimal constant price to maximize the gross profit:

$$
\text { Maxmize } \sum_{t=1}^{T}[P-c(t)] \cdot d(t) \text {. }
$$

The demand is defined as (2). Thus, the model is

$$
\begin{array}{ll}
\text { Maxmize } & \sum_{t=1}^{T}[P-c(t)] \cdot d(t) \\
\text { Subject to } & d(t)=n(t)+\min \left\{1, \lambda e^{\beta\left(P / P_{0}-1\right)}\right\} N(t-1), \\
& t=2, \ldots, T \\
& n(t) \\
& =\left[p+\frac{q}{m} N(t-1)\right][m-N(t-1)] e^{\beta\left(P / P_{0}-1\right)}, \\
& \\
& \\
n(t)=\sum_{\tau=1}^{t} n(t), \quad t=1, \ldots, T & t=2, \ldots, T \\
& d(1)=p m e^{\beta\left(P / P_{0}-1\right)}
\end{array}
$$

The sales division solves the optimal price $P^{*}$ and thus determines the demand $d(t)=d\left(t, P^{*}\right)$.

In the second stage, the purchasing division makes ordering decisions to minimize the total cost:

$$
\sum_{t=1}^{T}[k(t) \cdot v(t)+c(t) \cdot z(t)+h(t) \cdot x(t)]
$$

The total cost at period $t$ includes the total order cost $k(t)$. $v(t)+c(t) \cdot z(t)$ and the inventory holding cost $h(t) \cdot x(t)$.

The inventory balance equation is

$$
\begin{aligned}
x(t)=x(t-1)+z(t-1)-d\left(t-1, P^{*}\right) & \\
t & \\
t & =2, \ldots, T-1,
\end{aligned}
$$

where $d\left(t-1, P^{*}\right)$ is the demand at period $t-1$ given price $P^{*}$. We further assume that there is no inventory at the beginning of the planning horizon:

$$
x(0)=0 .
$$

Besides, both inventory level and order size are nonnegative:

$$
\begin{aligned}
& x(t) \geq 0, \quad t=1, \ldots, T, \\
& z(t) \geq 0, \quad t=1, \ldots, T .
\end{aligned}
$$

Thus, the purchasing division solves the following model:

$$
\begin{array}{ll}
\text { Minimize } & \sum_{t=1}^{T}[k(t) \cdot v(t)+c(t) \cdot z(t)+h(t) \cdot x(t)] \\
\text { Subject to } & x(t)=x(t-1)+z(t-1)-d\left(t-1, P^{*}\right), \\
& \\
& x(0)=0 \\
& x(t) \geq 0, \quad t=1, \ldots, T \\
& z(t) \geq 0, \quad t=1, \ldots, T .
\end{array}
$$

The decision variable of this model is the order size $z(t)$, $v(t)$, and $x(t)$.

3.5. Joint Decision Model. In the joint decision model, the firm considers pricing and inventory decisions simultaneously to maximize the profit:

$$
\text { Maximize } \sum_{t=1}^{T}[P \cdot d(t)-k(t) \cdot v(t)-c(t) \cdot z(t)-h(t) \cdot x(t)] \text {. }
$$

Therefore, the model is

$$
\begin{aligned}
\text { Maximize } & \sum_{t=1}^{T}[P \cdot d(t)-k(t) \cdot v(t)-c(t) \cdot z(t)-h(t) \cdot x(t)] \\
\text { Subject to } \quad x(t) & =x(t-1)+z(t-1)-d(t-1), \quad t=1, \ldots, T \\
d(t) & =n(t)+\min \left\{1, \lambda e^{\beta\left(P / P_{0}-1\right)}\right\} N(t-1), \\
n(t) & =\left[p+\frac{q}{m} N(t-1)\right][m-N(t-1)] e^{\beta\left(P / P_{0}-1\right)}, \\
& \\
N(t) & =\sum_{\tau=1}^{t} n(t), \quad t=2, \ldots, T \\
n(1) & =p m e^{\beta\left(P / P_{0}-1\right)} \\
& d(1)=p m e^{\beta\left(P / P_{0}-1\right)} \\
x(0) & =0 \\
x(t) & \geq 0, \quad t=1, \ldots, T \\
& z(t) \geq 0, \quad t=1, \ldots, T .
\end{aligned}
$$

The decision variables of this model are the price $P$ and $v(t), z(t)$ at each period $t$. 


\section{Algorithm}

We denote $c_{\min }=\min [c(t)]$ to be the minimum unit order cost over the planning horizon and give an upper and lower bound for the optimal price $P^{*}$ by the following lemma.

Lemma 1. For both the two-stage and joint decision models, the optimal price $P^{*}$ satisfies $c_{\min }<P^{*}<P_{0}[1-\ln (m T) / \beta]$.

Proof. The firm charges a price at least to make net profit; that is, $\pi>0$. On one hand, the price must cover all costs including unit order cost; that is, $P^{*}>c_{\min }$. On the other hand, the price should ensure the existence of demand; that is, the number of cumulative adopters $\sum_{t=1}^{T} n(t) \geq 1$. Since

$$
\begin{aligned}
& \sum_{t=1}^{T} n(t) \\
& =\sum_{t=2}^{T}\left[p+\frac{q}{m} N(t-1)\right][m-N(t-1)] e^{\beta\left(P / P_{0}-1\right)} \\
& \quad+p m e^{\beta\left(P / P_{0}-1\right)}<\sum_{t=2}^{T} m e^{\beta\left(P / P_{0}-1\right)}+m e^{\beta\left(P / P_{0}-1\right)} \\
& =m T e^{\beta\left(P / P_{0}-1\right)}
\end{aligned}
$$

we have $m T e^{\beta\left(P / P_{0}-1\right)}>\sum_{t=1}^{T} n(t) \geq 1$ and thus obtain $P^{*}<P_{0}[1-\ln (m T) / \beta]$. Therefore, the optimal price $P^{*}$ satisfies $c_{\min }<P^{*}<P_{0}[1-\ln (m T) / \beta]$ for both the two-stage and joint decision models. Considering the complexity of the new product pricing problems [16], we develop heuristic algorithms for both models.

4.1. Algorithms for the Two-Stage Decision Model. In the twostage decision model, the firm makes pricing and ordering decisions by two steps. First, the sales division finds an optimal price $P^{*}$ to maximize the gross profit $\pi_{g}=\sum_{t=1}^{T}[P-$ $c(t)] d(t)$ by solving model (4). Second, the purchasing division determines an optimal ordering decision to minimize the total cost by solving model (9). We now give algorithms for models (4) and (9).

We use the following Gaussian Random-Walk search algorithm to solve model (4):

(1) Start with $i=1$, an initial price $P_{i}$, and calculate the gross profit $\pi_{g}\left(P_{i}\right)$ given the demand $d(t)=d\left(P_{i}, t\right)$. Set the initial best price $P^{*}=P_{i}$ and the initial best gross profit $\pi_{g}^{*}=\pi_{g}\left(P_{i}\right)$.

(2) Draw $P_{i+1}=P_{i}+\epsilon, \epsilon \sim N\left(0, s^{2}\right)$. Jump to Step 3 if $c_{\min }<P_{i+1}<P_{0}[1-\ln (m T) / \beta]$; otherwise, repeat Step 2.

(3) Calculate the gross profit $\pi_{g}\left(P_{i+1}\right)$ given the demand $d(t)=d\left(P_{i+1}, t\right)$.

(4) If $\pi_{g}\left(P_{i+1}\right) / \pi_{g}^{*}>1$, update the best price $P^{*}=P_{i+1}$, the best gross profit $\pi_{g}^{*}=\pi_{g}\left(P_{i+1}\right)$, reset $i=1, P_{i}=$ $P^{*}$, and jump to Step 2. Otherwise, jump to Step 5.
(5) Compute $\alpha=\min \left\{1, \pi_{g}\left(P_{i+1}\right) / \pi_{g}\left(P_{i}\right)\right\}$.

(6) Consider $i=i+1 . P_{i}=P_{i}$ with probability $\alpha$ (move) and one has $P_{i}=P_{i-1}$ with probability $1-\alpha$ (stay). Update $\pi_{g}\left(P_{i}\right)$ accordingly.

(7) Stop if $i=N$. Otherwise, jump to Step 2.

This algorithm stops if the best gross profit $\pi_{g}^{*}$ and the best price $P^{*}$ have not been improved after $N$ random walks. In Step 6, $P_{i}$ transits to $P_{i+1}$ if $\pi_{g}\left(P_{i+1}\right) \geq \pi_{g}\left(P_{i}\right)$. Even if $\pi_{g}\left(P_{i+1}\right)<\pi_{g}\left(P_{i}\right), P_{i}$ transits to $P_{i+1}$ with probability $\alpha$. This mechanism automatically seeks out price areas with high gross profit and makes the price walk freely in the interval $\left(c_{\min }, P_{0}[1-\ln (m T) / \beta]\right)$. We choose $N=1000, s=2$, after experiment trials in our numerical case.

We solve model (9) by the Wagner-Whitin algorithm, a classic dynamic lot sizing algorithm proposed by Wagner and Whitin [17].

4.2. Algorithm for the Joint Decision Model. Since $v(t)$ indicates whether to replenish product at period $t$, we term it as "order policy" to facilitate our algorithm development. Given any price $P$, we are able to know the demand $d(t)=$ $d(t, P)$ and thus model (11) becomes the standard dynamic lot sizing model, which could be solved by the Wagner-Whitin algorithm [17].

We denote the order policy under price $P$ as $\mathbf{v}(P)$, considering that we can determine the uniquely optimal order policy $v(t)$ for any given $P$. Assume that the firm charges price $P^{\prime}$ but uses order policy $\mathbf{v}(P)$, the optimal order policy under price $P$. In this case, we can easily solve the order size at each period $z(t)$. We denote $\pi\left(\mathbf{v}(P), P^{\prime}\right)$ as the profit for charging price $P^{\prime}$ but use order policy $\mathbf{v}(P)$.

Then, we propose the following algorithm to solve model (11):

(1) Set $i=1$ and select an initial price, $P_{i}$.

(2) Calculate the demands given by $d\left(t, P_{i}\right)$ for $t=$ $1,2, \ldots, N$. Use the Wagner-Whitin algorithm to determine an optimal ordering policy $\mathbf{v}\left(P_{i}\right)$ and the profit $\pi\left(\mathbf{v}\left(P_{i}\right), P_{i}\right)$

(3) For the ordering policy $\mathbf{v}\left(P_{i}\right)$ derived in Step 2, use a Gaussian Random-Walk algorithm to find a price $P_{i+1}\left(c_{\min }<P_{i+1}<P_{0}[1-\ln (m T) / \beta]\right)$ to maximize $\pi\left(\mathbf{v}\left(P_{i}\right), P_{i+1}\right)$.

(4) Calculate the profit increase $\Delta \pi=\pi\left(\mathbf{v}\left(P_{i}\right), P_{i+1}\right)-$ $\pi\left(\mathbf{v}\left(P_{i}\right), P_{i}\right)$. Set $i=i+1$.

(5) Stop if $\Delta \pi<\xi$, where $\xi$ is a small enough positive number; otherwise, jump to Step 2.

Note that the firm should determine both the price and the ordering decisions. Our algorithm keeps one decision (price or ordering policy) unchanged and improves the profit $\pi$ by varying another decision. Step 3 keeps the order policy $\mathbf{v}\left(P_{i}\right)$ constant and varies the price to improve the profit $\pi\left(\mathbf{v}\left(P_{i}\right), \cdot\right)$. Similarly, Step 2 keeps the price $P_{i}$ constant and adjusts the order policy to improve the profit $\pi\left(\cdot, P_{i}\right)$. Thus, every iteration in the algorithm helps improve the profit $\pi$. 
TABLE 1: Comparison of the optimal pricing and ordering decisions.

\begin{tabular}{|c|c|c|c|c|c|c|c|c|c|c|c|c|}
\hline & $t=1$ & $t=2$ & $t=3$ & $t=4$ & $t=5$ & $t=6$ & $t=7$ & $t=8$ & $t=9$ & $t=10$ & $t=11$ & $t=12$ \\
\hline \multicolumn{13}{|c|}{ Two-stage decision model: $P^{*}=47.5, \pi=188320$} \\
\hline$v(t)$ & 1 & 0 & 0 & 1 & 0 & 1 & 1 & 1 & 1 & 0 & 1 & 0 \\
\hline$z(t)$ & 2529 & 0 & 0 & 5512 & 0 & 4062 & 4298 & 4077 & 7617 & 0 & 7480 & 0 \\
\hline$n(t)$ & 374 & 629 & 1011 & 1500 & 1946 & 2022 & 1502 & 720 & 226 & 55 & 12 & 2 \\
\hline$N(t)$ & 374 & 1003 & 2014 & 3514 & 5460 & 7482 & 8984 & 9704 & 9930 & 9985 & 9997 & 9999 \\
\hline$d(t)$ & 374 & 769 & 1386 & 2253 & 3259 & 4062 & 4298 & 4077 & 3852 & 3765 & 3743 & 3737 \\
\hline \multicolumn{13}{|c|}{ Joint decision model: $P^{*}=52.1, \pi=221860$} \\
\hline$v(t)$ & 1 & 0 & 0 & 1 & 0 & 1 & 0 & 1 & 1 & 0 & 1 & 0 \\
\hline$z(t)$ & 1757 & 0 & 0 & 3457 & 0 & 5890 & 0 & 3412 & 6549 & 0 & 6087 & 0 \\
\hline$n(t)$ & 297 & 459 & 689 & 988 & 1318 & 1577 & 1617 & 1350 & 890 & 469 & 209 & 84 \\
\hline$N(t)$ & 297 & 756 & 1445 & 2433 & 3751 & 5328 & 6945 & 8295 & 9185 & 9654 & 9863 & 9947 \\
\hline$d(t)$ & 297 & 547 & 913 & 1417 & 2040 & 2691 & 3199 & 3412 & 3353 & 3196 & 3075 & 3012 \\
\hline
\end{tabular}

The stop rule is that there is no improvement in profit $\Delta \pi=0$ (i.e., $\Delta \pi<\xi$, where $\xi=10^{-6}$ in Step 5). The Gaussian Random-Walk search algorithm in Step 3 is very similar to that in the two-stage decision model, and we only need to replace $\pi_{g}(\cdot)$ as $\pi\left(\mathbf{v}\left(P_{i}\right), \cdot\right)$.

From a practical viewpoint, the price can be only integer multiples of the smallest unit of currency. Therefore, we can also search all integer multiples of the smallest currency unit between $c_{\min }$ and $P_{0}[1-\ln (m T) / \beta]$ and find the optimal price. It reports very similar results with Gaussian RandomWalk search algorithm, suggesting the reliability of the latter one. Thus, we only report results obtained from the Gaussian Random-Walk search algorithm in this paper.

\section{Numerical Case}

We choose a packaged imported food item called "Zespri Kiwifruit" from Netherlands provided by an online fresh produce retailer in Central China. The unit order cost $c(t)=$ 30 (the currency unit is Chinese Yuan Renminbi (CNY) in our paper), setup cost $k(t)=40000$, unit holding cost $h(t)=$ 10 , and the number of periods $T=12$. The retailer charges a regular price $P_{0}=60$, and the repeat-purchase rate $\lambda=.2$, the market potential $m=10000$, and the price parameter $\beta=-3$. We set external influence $p=.02$ and internal influence $q=.4$ as suggested by Sultan et al. [4].

In the two-stage decision model, the sales division determines an optimal constant price $P^{*}$ to maximize the gross profit by solving model (4). Then, the purchasing division determines an optimal ordering decision given demand $d\left(t, P^{*}\right)$ to minimize total cost by solving model (9). We use these results as a baseline to evaluate potential benefits of the joint decision model, in which the firm determines pricing and ordering decisions simultaneously by solving model (11).

5.1. Pricing and Ordering Decisions for Both Models. We report the optimal pricing and ordering decisions for both models in Table 1 . The firm charges the optimal price $P^{*}=$ 47.5 and earns profit $\pi=188320$ for the two-stage decision model and charges the optimal price $P^{*}=52.1$ and earns profit $\pi=221860$ for the joint decision model. Besides, the order policy $v(t)$ of the two models is also different: the firm replenishes this kind of repeat-purchase product at $t=7$ in the two-stage decision model, but it does not in the joint decision model.

Joint decision model evaluates all costs and revenue and thus finds a better way to price and order this kind of imported food. Comparing to the two-stage decision, joint ordering and pricing decisions bring in substantially extra profit (as large as $17.81 \%$ ). Since the two-stage decision model maximizes the gross profit, the sales division tends to charge a lower price to increase the sales volume. We find that both the number of new adopters $n(t)$ and the demand $d(t)$ are larger for two-stage decision model. However, the increase in sales volume does not ensure profitability. In consequence, cooperation between the sales division and the purchasing division turns out valuable.

Since the diffusion process and repeat-purchase rate are important for repeat-purchase products, we also conduct sensitivity analysis for these two factors and examine their effects on the optimal price $P^{*}$ and the profit $\pi$.

5.2. The Influences of $q / p$. The internal influence $q$, the external influence $p$, and the price $P$ jointly determine the diffusion process of repeat-purchase products when the market potential $m$ and the price parameter $\beta$ keep constant (see (1)). Consumers show initiative to adopt some repeatpurchase products, but they become hesitant to adopt others. That is, the internal influence $q$ and the external influence $p$ vary from different repeat-purchase products. Particularly, $q / p$ has large variation for different products while $p+q$ does not [4]. In consequence, we keep $p+q$ constant and examine the effects of $q / p$.

We show how the optimal price $P^{*}$ and the profit $\pi$ vary with $q / p$ in Figure 1. We observe that joint decision model makes the firm charge higher prices and earn more profits than two-stage decision model for all $q / p$. Since joint decision model takes all kinds of costs rather than only unit order cost into consideration, the firm has less space to offer low prices. However, cooperation between the sales division and 


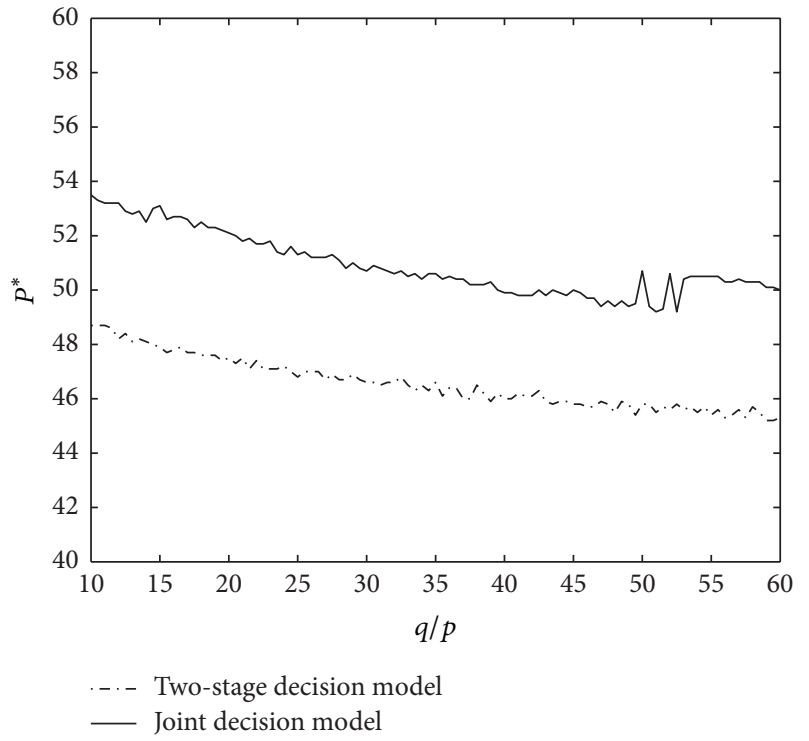

(a) $P^{*} \sim q / p$

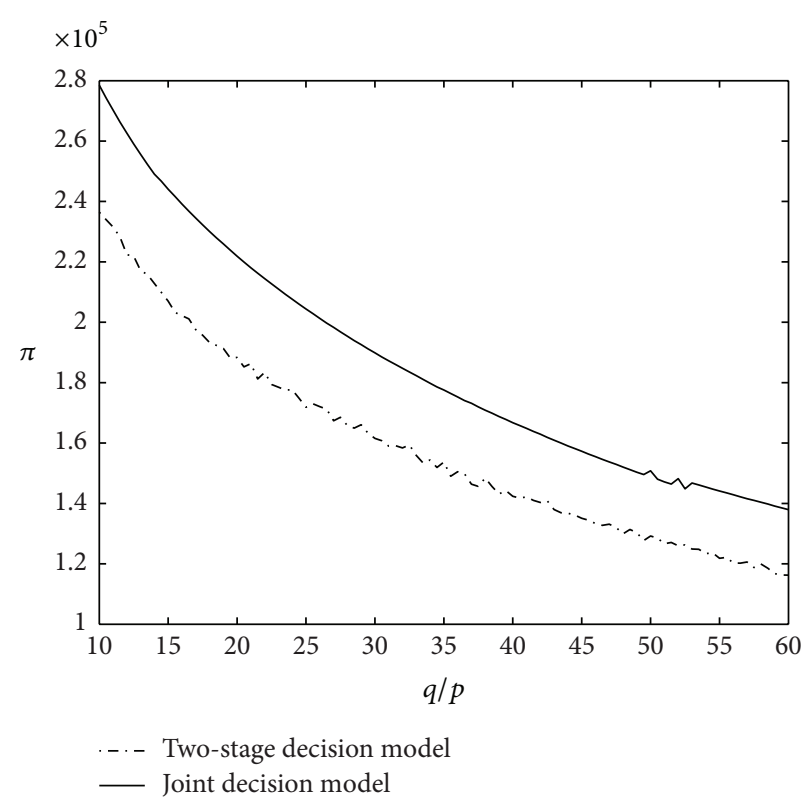

(b) $\pi \sim q / p$

Figure 1: The influences of $q / p$.

the purchasing division makes the pricing and ordering decisions wiser and thus obtain more profits.

Besides, both the optimal price $P^{*}$ and the profit $\pi$ show a decreasing trend with $q / p$ for both models. When the ratio of the internal influence to the external influence $q / p$ increases, consumers in the market show less innovativeness and more imitativeness. Thus, the firm should charge low prices to promote the product diffusion process and results in profit reduction.

5.3. The Influences of Repeat-Purchase Rate $\lambda$. Repeatpurchase rate varies from different kinds of repeat-purchase products. Various factors such as reputation of the firm, product quality, service quality, and demographic variables may influence the repeat-purchase rate $\lambda$. Since both $\lambda$ and the price $P$ influence the demand from cumulative adopters, the firm should understand how to adjust prices with regard to the repeat-purchase rate.

We show how the optimal price $P^{*}$ and the profit $\pi$ vary with $\lambda$ in Figure 2 . We find that joint decision model makes the firm charge higher or at least of the same prices and earn more profits than two-stage decision model for all $\lambda$. Similarly, we contribute this observation to the value of cooperation between the sales and the purchasing divisions.

Interestingly, the optimal price $P^{*}$ decreases first, increases later, and keeps constant eventually with $\lambda$ for both two-stage and joint decision models. First, when the repeat-purchase rate is low (i.e., $\lambda<.5$ in Figure 2(a)), the firm should reduce prices with the increase of $\lambda$. Low prices can increase both the number of new adopters and the number of cumulative adopters who show repeat-purchase behavior, and the latter effect becomes greater when the repeat-purchase rate increases. Thus, the optimal price $P^{*}$ decreases with $\lambda$ when repeat-purchase rate is low. Second, when the repeat-purchase rate is fairly high (i.e., $.5<\lambda<.7$ in Figure 2(a)), the firm should increase prices with the increase of $\lambda$. Since the high repeat-purchase rate and low prices can at most make all cumulative adopters purchase this kind of imported food, the firm does not need to lower prices when $\lambda e^{\beta\left(P / P_{0}-1\right)} \geq 1$. Instead, the firm will increase prices with $\lambda$ when the repeat purchase is fairly high. Finally, when the repeat-purchase rate is very high (i.e., $\lambda>.7$ in Figure 2(a)), the firm will keep a constant, high price. The firm does not increase prices again because higher prices would shapely reduce the number of new adopters. Thus, they keep a constant, high price in this case.

The profit $\pi$ increases at first and keeps constant eventually with $\lambda$. When the repeat-purchase rate is not very high (i.e., $\lambda<.7$ in Figure $2(\mathrm{~b})$ ), the profit increases with $\lambda$ since the repeat-purchase behavior of the cumulative adopters increases. When repeat-purchase rate is very high (i.e., $\lambda>.7$ in Figure 2(b)), however, the profit keeps constant since all cumulative adopters purchase this kind of imported food.

\section{Concluding Remarks}

This paper studies ordering and pricing problems for new repeat-purchase products. We incorporate the repeatpurchase rate and price effects into the Bass model to characterize the demand of repeat-purchase products. We consider both two-stage and joint decision models, apply them to a specific imported food provided by an online fresh produce retailer in Central China, and solve them by Gaussian Random-Walk and Wagner-Whitin based algorithms.

Three results are worthy of attention. First, joint pricing and ordering decisions bring more significant profits than 


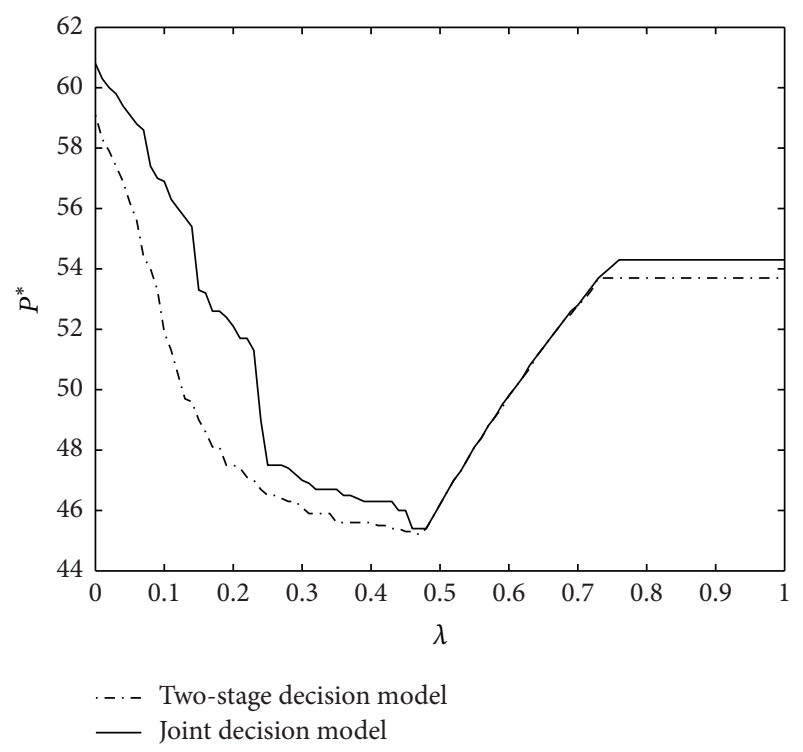

(a) $P^{*} \sim \lambda$

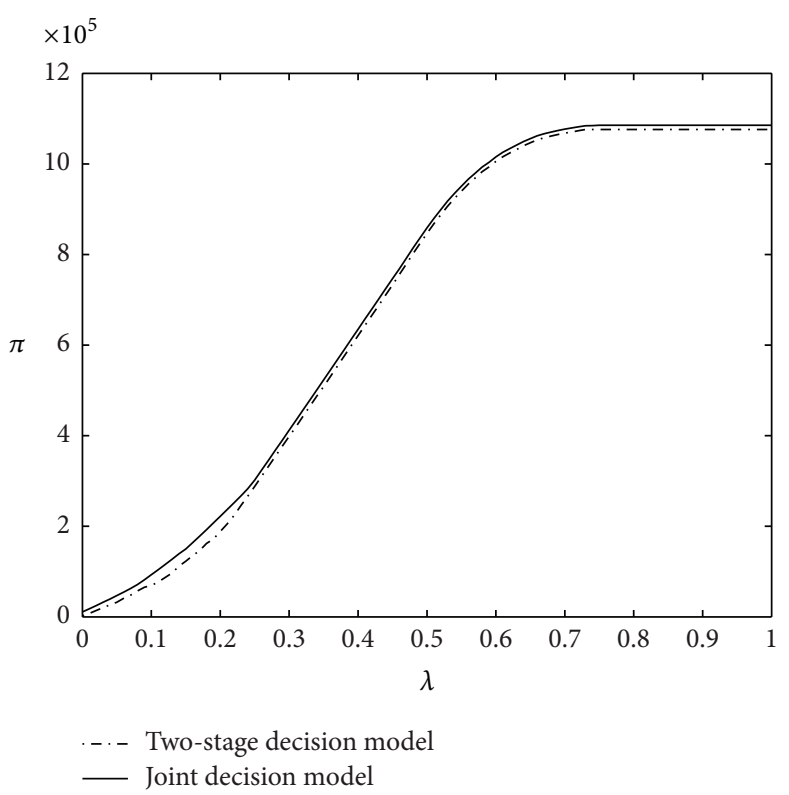

(b) $\pi \sim \lambda$

FIGURE 2: The influences of repeat-purchase rate $\lambda$.

making pricing and ordering decisions sequentially (as large as 17.81 percent in our case). Second, a great initiative in adoption significantly increases price premium and profit. When the ratio of the internal influence to the external influence increases, both th price and the profit decrease. Thus, the firm should make efforts to increase the initiative in adoption. Finally, the optimal price shows a U-shape (i.e., decreases first and increases later) relationship and the profit increases gradually with the repeat-purchase rate when it is still not very high (i.e., $\lambda<.7$ ). Thus, it is worthwhile for the firm to improve repeat-purchase rate to a fairly high level.

\section{Conflict of Interests}

The authors declare that there is no conflict of interests regarding the publication of this paper.

\section{Acknowledgment}

The financial support from the National Natural Science Foundation Program of China [no. 71271095] is gratefully acknowledged.

\section{References}

[1] F. M. Bass, "A new product growth for model consumer durables," Management Science, vol. 15, pp. 215-227, 1969.

[2] C. Stummer, E. Kiesling, M. Günther, and R. Vetschera, "Innovation diffusion of repeat purchase products in a competitive market: an agent-based simulation approach," European Journal of Operational Research, vol. 245, no. 1, pp. 157-167, 2015.

[3] B. Robinson and C. Lakhani, "Dynamic price models for newproduct planning," Management Science, vol. 21, no. 10, pp. 1113$1122,1975$.
[4] F. Sultan, J. U. Farley, and D. R. Lehmann, "A meta-analysis of applications of diffusion models," Journal of Marketing Research, vol. 27, pp. 70-77, 1990.

[5] V. Mahajan, E. Muller, and F. M. Bass, "New product diffusion models in marketing: a review and directions for research," Journal of Marketing, vol. 54, no. 1, pp. 1-26, 1990.

[6] F. M. Bass, T. V. Krishnan, and D. C. Jain, "Why the bass model fits without decision variables," Marketing Science, vol. 13, no. 3, pp. 203-223, 1994.

[7] Ö. Bilginer and F. Erhun, "Production and sales planning in capacitated new product introductions," Production and Operations Management, vol. 24, no. 1, pp. 42-53, 2015.

[8] S. Bhattacharya, V. D. R. Guide Jr., and L. N. Wassenhove, "Optimal order quantities with remanufacturing across new product generations," Production and Operations Management, vol. 15, no. 3, pp. 421-431, 2006.

[9] C. A. Yano and S. M. Gilbert, "Coordinated pricing and production/procurement decisions: a review," in Managing Business Interfaces, pp. 65-103, Springer, 2004.

[10] L. Lu, J. Zhang, and W. Tang, "Optimal dynamic pricing and replenishment policy for perishable items with inventory-leveldependent demand," International Journal of Systems Science, pp. 1-15, 2014.

[11] C.-T. Yang, L.-Y. Ouyang, and H.-H. Wu, "Retailer's optimal pricing and ordering policies for non-instantaneous deteriorating items with price-dependent demand and partial backlogging," Mathematical Problems in Engineering, vol. 2009, Article ID 198305, 18 pages, 2009.

[12] R. Maihami and I. N. Kamalabadi, "Joint pricing and inventory control for non-instantaneous deteriorating items with partial backlogging and time and price dependent demand," International Journal of Production Economics, vol. 136, no. 1, pp. 116122,2012 
[13] Q. Feng, S. Luo, and D. Zhang, "Dynamic inventory-pricing control under backorder: demand estimation and policy optimization," Manufacturing \& Service Operations Management, vol. 16, no. 1, pp. 149-160, 2014.

[14] H. Fu, B. Dan, and X. Sun, "Joint optimal pricing and ordering decisions for seasonal products with weather-sensitive demand," Discrete Dynamics in Nature and Society, vol. 2014, Article ID 105098, 8 pages, 2014.

[15] P. Berling and V. Martínez-de-Albéniz, "Optimal inventory policies when purchase price and demand are stochastic," Operations Research, vol. 59, no. 1, pp. 109-124, 2011.

[16] T. V. Krishnan, F. M. Bass, and D. C. Jain, "Optimal pricing strategy for new products," Management Science, vol. 45, no. 12, pp. 1650-1663, 1999.

[17] H. M. Wagner and T. M. Whitin, "Dynamic version of the economic lot size model," Management Science, vol. 5, no. 1, pp. 89-96, 1958. 


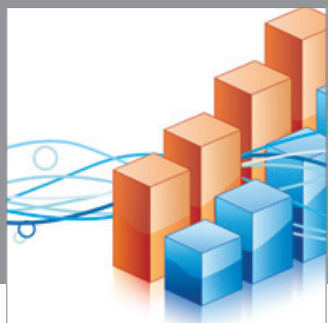

Advances in

Operations Research

mansans

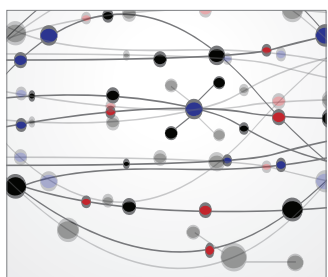

The Scientific World Journal
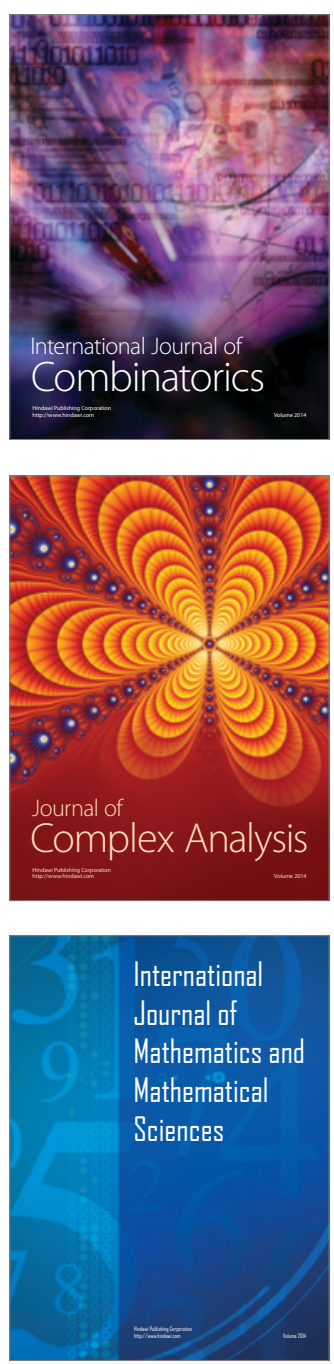
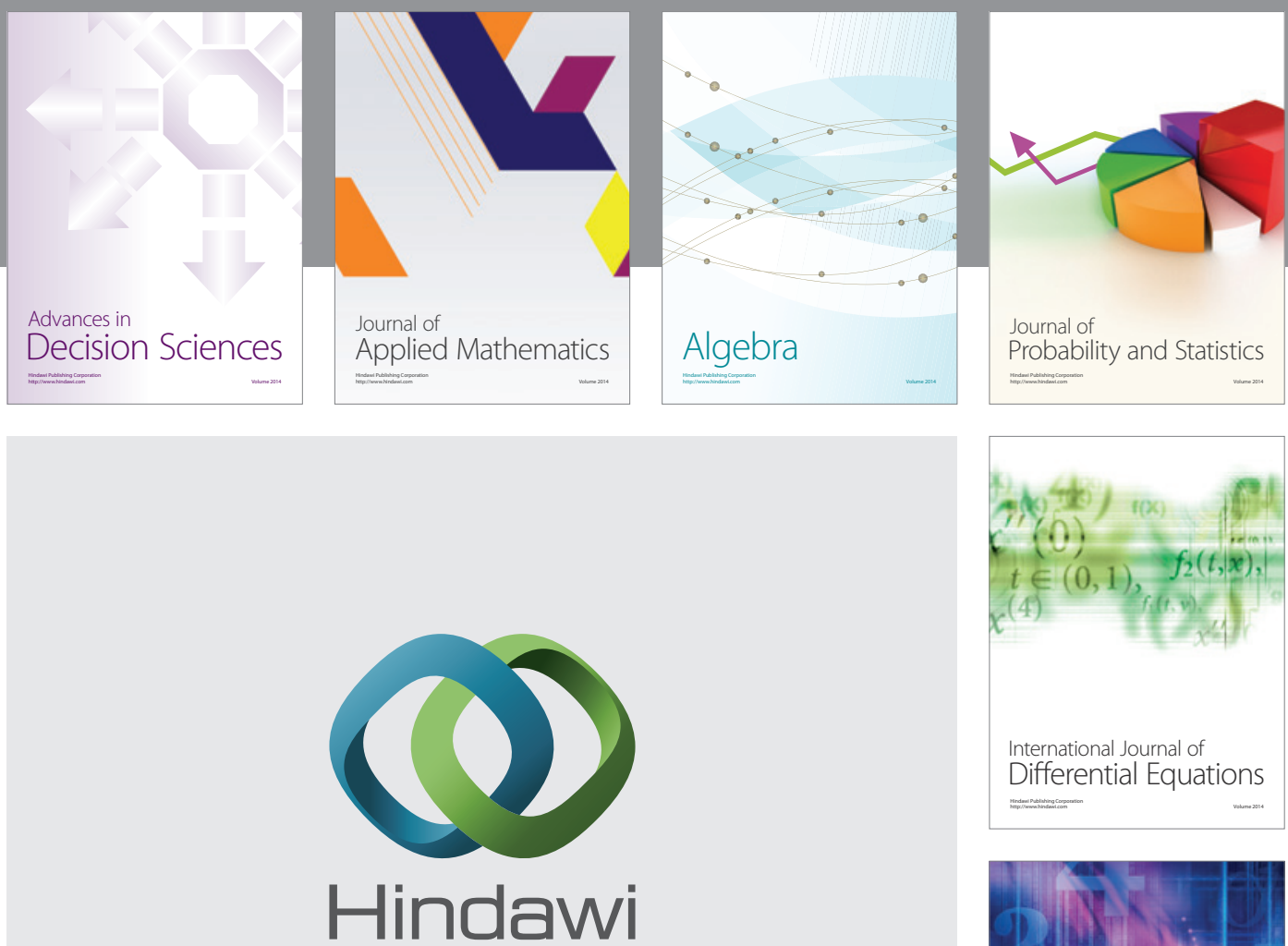

Submit your manuscripts at http://www.hindawi.com
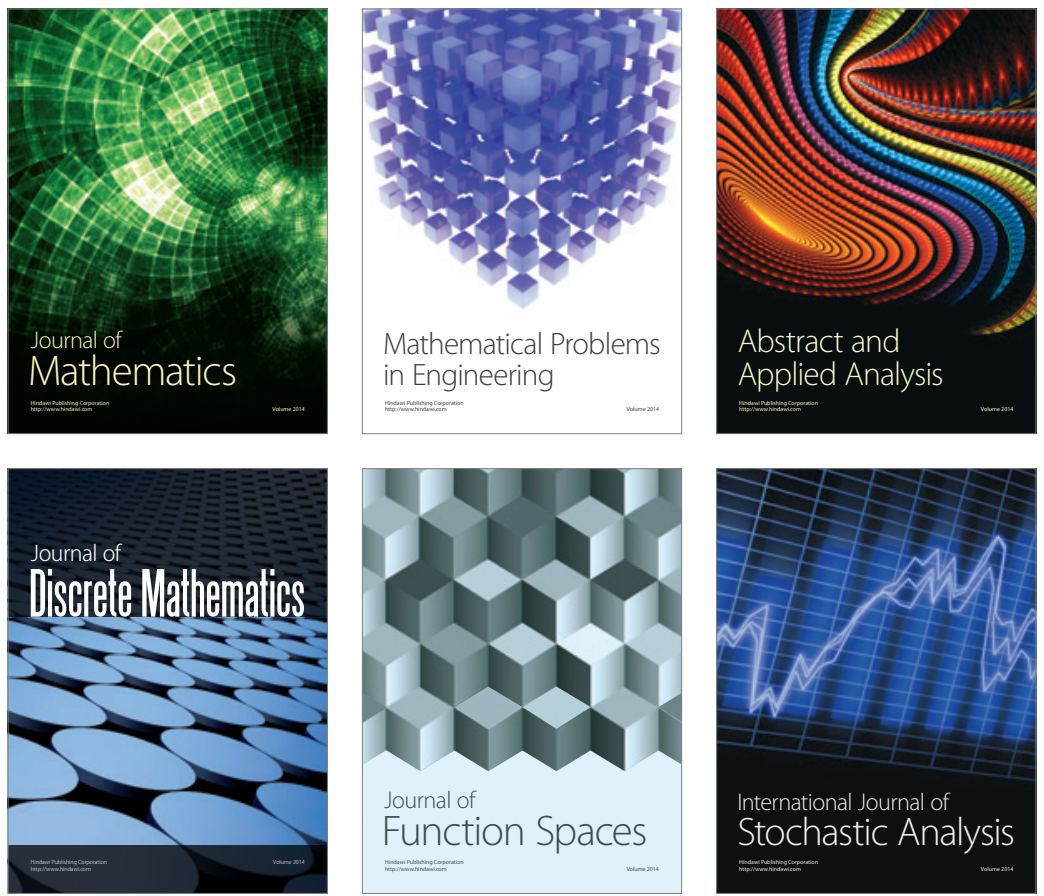

Journal of

Function Spaces

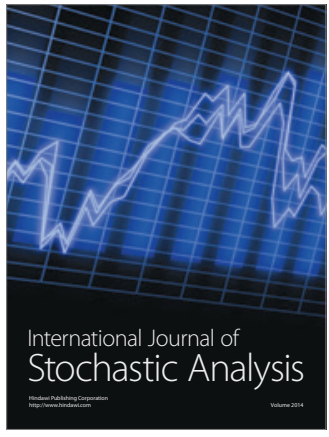

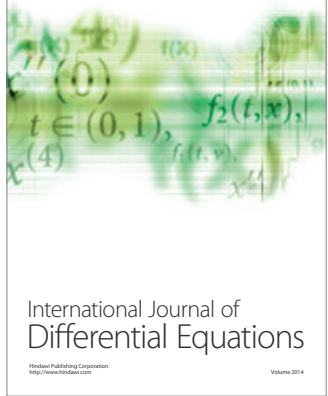
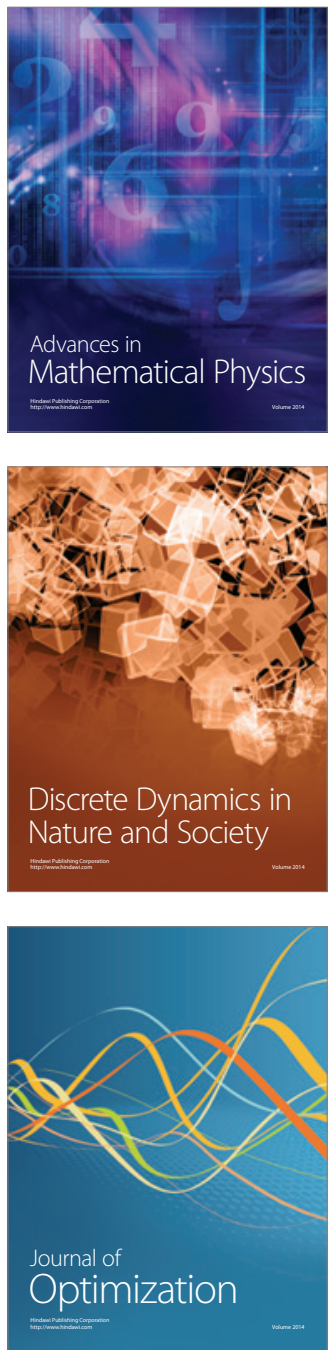